\title{
La Communauté économique européenne et la Commission économique pour l'Afrique de l'ONU: la difficile convergence de deux projets de développement pour le continent africain (1958-1963)
}

\section{Guia MIGANI}

Au début des années soixante, l'Afrique commence véritablement à faire partie du système international comme un acteur à part entière. ${ }^{1}$ Elle n'est plus un continent soustrait - presque entièrement - aux dynamiques mondiales par son appartenance aux empires coloniaux. ${ }^{2}$ Dans ce contexte, on assiste à une double tendance. D'une part l'Afrique s'ouvre aux forces politiques et économiques internationales: les Etats africains nouvellement indépendants veulent être admis aux Nations Unies et n'hésitent pas à faire jouer la concurrence entre le monde occidental et le bloc communiste pour se procurer des aides économiques toujours plus consistantes. Ils entrent au FMI et à la Banque mondiale, et recherchent des nouveaux partenaires économiques. D'autre part on assiste à une certaine régionalisation des affaires africaines: les Etats africains non seulement revendiquent le droit de gérer en pleine autonomie leurs affaires intérieures, mais ils cherchent aussi à coordonner leurs politiques, à créer un marché intégré et à élaborer un projet commun de développement économique et social.

La Commission économique pour l'Afrique (CEA), un organisme régional des Nations Unies, est pleinement représentative de cette double tendance: elle représente, pour les Etats africains, le lieu privilégié où on se fait entendre sur les questions d'intérêt mondial. Cependant la CEA est aussi un organisme régional dont on veut, peu à peu, exclure les pays européens qui en sont membres.

Cet article propose une analyse du débat qui se développe au sein de la CEA entre les pays membres de la CEE et les Etats africains entre 1958, date de la création de la CEA, et 1963, avec la naissance de l'Organisation de l'Unité

1. L'auteur remercie le professeur Gérard Bossuat pour son soutien dans la rédaction de cet article, qui a fait l'objet d'une communication lors d'une table ronde organisée par l'Université de Cergy Pontoise sur «L'originalité des Communautés européennes dans le processus de mondialisation» le 19 septembre 2005.

2. Cf. M. MICHEL, Décolonisation et émergence du Tiers Monde, Hachette, Paris, 1993; C. COQUERY-VIDROVITCH, Afrique noire. Permanences et ruptures, L'Harmattan, Paris, 1992; M. MEREDITH, The state of Africa. A History of fifty years of independence, Free Press, London, 2005; E. M'BOKOLO, L'Afrique du XXème siècle. Le continent convoité, Seuil, Paris, 1985. C. COKER, Nato, the Warsaw Pact and Africa, Macmillan, London, 1985; F. DURPAIRE, Les Etats-Unis ont-ils décolonisé l'Afrique noire francophone?, L'Harmattan, Paris, 2005; C. ANDREW, V. MITROKIN, The world was going our way: the KGB and the battle for the Third World, Basic Books, New York, 2005; R.C. NATION, M. KAUPPI, The Soviet impact in Africa, Lexington Books, Lexington, 1984; O.A. WESTAD, The Global Cold War: Third World Interventions And The Making Of Our Times, Cambridge University Press, Cambridge, 2005. 
Africaine (OUA) et la signature de la Convention de Yaoundé. ${ }^{3}$ Au sein de la CEA on rencontre plusieurs acteurs qui se confrontent sur le problème du développement de l'Afrique, sur la place de l'Afrique dans le système international - les deux questions étant très étroitement liées - et sur le rôle de la CEE en Afrique. Parmi ces acteurs, on peut identifier les pays africains indépendants (partagés entre Etats arabes et Etats de l'Afrique sub-saharienne), les Etats associés à la CEE qui entrent dans la CEA après être devenus indépendants et les puissances coloniales européennes. On a cherché ainsi à étudier quel type d'échange il y a eu entre ces groupes d'acteurs et de voir si cette confrontation a mené vers quelques résultats.

Les Six ne font pas tous partie de la CEA puisque la participation à la Commission régionale est réservée aux pays africains indépendants et aux Etats responsables pour des territoires africains. Au moment de sa création sont ainsi membres de la CEA seulement la Belgique, la France et l'Italie. Cependant, si les Six ne sont pas tous membres de Commission économique pour l'Afrique, tous contribuent à l'élaboration de positions communes qui sont défendues lors des sessions de la CEA. Parmi les membres européens de la CEA il y a aussi la Grande Bretagne, l'Espagne et le Portugal. Toutefois, dans ce cadre on se concentrera sur les Six et les Etats africains.

\section{La naissance de la CEA. Les Etats africains découvrent un nouvel instrument de pression contre les Six}

La CEA a été créée par une résolution du Conseil Economique et Social le 29 avril 1958, en exécution d'une résolution de l'Assemblée générale de l'ONU. L'idée d'une commission économique pour l'Afrique n'était pas nouvelle, mais elle s'était toujours heurtée à l'hostilité des puissances coloniales, peu soucieuses d'impliquer davantage les Nations Unies dans les territoires sous leur contrôle. Au cours des années cinquante, la situation s'était partiellement modifiée à la suite des changements politiques intervenus en Afrique, et de l'attitude plus libérale adoptée par les puissances coloniales. Par ailleurs, l'importance grandissante des pays du Tiers Monde aux Nations Unies avait permis de surmonter les dernières réticences.

Au moment de sa création la Commission compte parmi ses membres dix Etats africains indépendants, ${ }^{4}$ six puissances européennes ayant des territoires africains, ${ }^{5}$

3. Cet article est fondé sur la consultation de plusieurs archives: les Archives Historiques de l'Union Européenne [AHUE], les archives du Ministère des Affaires Etrangères français [MAEF], le Centre historique des Archives nationales françaises [CHAN], et les Archives Nationales des Etats-Unis [NARA-Washington].

4. Ethiopie, Ghana, Guinée, Libéria, Libye, Maroc, République Arabe Unie, Soudan, Tunisie, Union Sud-Africaine.

5. Belgique, Espagne, France, Grande Bretagne, Italie, Portugal. 
et huit territoires associés qui participent sans droit de vote à toutes les réunions de la Commission. ${ }^{6}$ Selon les termes de son mandat, la CEA doit:

- prendre des mesures et participer à leur exécution pour faciliter une action concertée en vue du développement et du renforcement des relations économiques des pays africains entre eux, ainsi qu'avec le reste du monde;

- procéder ou faire procéder à des enquêtes sur le problème du développement économique de l'Afrique;

- rassembler ou faire rassembler toute documentation concernant les problèmes posés par l'évolution économique des territoires africains et en assurer la diffusion;

- fournir des services consultatifs aux pays africains qui en feront la demande;

- aider à formuler des politiques coordonnées pour favoriser le développement économique et technologique de la région. ${ }^{7}$

Dans l'exercice de ses compétences, la CEA doit tenir compte d'études préalables, et établir des relations avec les organisations qui ont une compétence sur l'Afrique. La CEA est ainsi portée à coopérer avec les institutions spécialisées des Nations Unies, avec la Commission de coopération technique en Afrique au Sud du Sahara $(\mathrm{CCTA})^{8}$ et, éventuellement, avec la CEE.

La première session de la CEA étant prévue pour janvier 1959, les Six s'efforcent de mettre à point une attitude commune pour défendre la Convention d'association des Territoires d'outremer (TOM) à la CEE, et faire admettre un représentant de la Commission européenne aux travaux de la CEA. Les Six redoutent de fortes critiques contre le régime d'association des TOM à la CEE. Celui-ci prévoit la création d'une zone de libre échange eurafricaine; cet objectif, toutefois, n'est pas mentionné dans les traités de Rome, ni dans la Convention d'association annexée au traité. Dans le traité sur la CEE les Six avaient seulement inscrit les principes de l'association: la promotion du développement économique et social des territoires associés et l'instauration de relations économiques étroites entre eux et la Communauté. Une convention d'application, annexée au traité, fixait les modalités de l'association pour une période de cinq ans, mais elle était renouvelable sur décision unanime du Conseil des ministres. La convention contenait des dispositions concernant le régime commercial, le droit d'installation

6. Gambie, Kenya et Zanzibar, Nigeria, Ouganda, Protectorat de Somalie, Sierra Leone, Tanganyika, Somalie sous administration italienne. Il s'agit de pays africains qui ne sont pas encore indépendants, et qui peuvent être associés aux travaux de la Commission sur demande de l'Etat responsable.

7. AHUE, CM 2/1059, dossier 1026, Annexe III au rapport au Conseil économique et social sur la première session de la CEA (29 décembre 1958-6 janvier 1959).

8. «La CCTA a été créée en 1950 par la France et le Royaume Uni pour répondre à un besoin d'échange d'informations sur un plan purement scientifique et technique. Ses membres ont été portés à neuf avec l'admission de la Guinée [...] (sont également membres la Belgique, le Portugal, l'Union Sud-Africaine, la Fédération de Rhodésie et du Nyassaland, le Liberia et le Ghana). La CCTA est une organisation intergouvernementale purement technique, dotée d'un budget modeste (2.000 millions). Son efficacité repose sur les mesures prises par les Gouvernements membres à la suite de ses recommandations qui doivent être adoptées à l'unanimité». CHAN, Fonds privé Foccart, dossier 103, Note à l'attention de M. le Président de la Communauté, sans date. 
dans les territoires africains et le Fonds d'investissement (FEDOM), destiné à financer des projets à caractère économique et social dans les territoires d'outremer. Il était aussi établi que les Six d'une part, et les TOM d'autre part, s'accordent des préférences mutuelles douanières et contingentaires. Sur le plan douanier, il ne devait pas subsister de discriminations à l'entrée dans les TOM entre les produits des cinq et ceux des métropoles. ${ }^{9}$ Les produits agricoles des TOM n'avaient pas droit au régime de la politique agricole commune; toutefois il leur était assuré un traitement préférentiel par la mise en place d'un Tarif Extérieur Commun (TEC), qui ne concernait pas les produits en provenance des Territoires d'outremer. ${ }^{10}$

En prévision de la première réunion de la CEA, qui se tiendra à Addis Abeba du 29 décembre 1958 au 6 janvier 1959, le Comité des Représentants Permanents (COREPER) de la CEE discute de la stratégie à adopter pour défendre le régime d'association des TOM à la CEE. Ce qui pose le plus de problèmes aux pays tiers est le TEC, qui sera imposé à leurs exportations mais qui, par contre, ne concernera pas les exportations des territoires associés. Les pays tiers, et en particulier les pays exportateurs de produits tropicaux, craignent de subir des fortes pertes sur les marchés européens.

Pendant la réunion, les Français sollicitent les Cinq pour élaborer des réponses communes aux attaques qui seront portées contre l'association des TOM à la CEE:

«L'expérience, non seulement du GATT, mais aussi des trois commissions régionales de l'ONU, ne laisse guère de doute à cet égard, non plus que le rôle que le Royaume Uni jouera dans ce concert de critiques». ${ }^{11}$

En particulier, Paris s'attend à deux types de reproches: celui visant le principe des préférences accordées aux pays associés en faveur desquels il y aura un détournement du trafic commercial, et celui affirmant l'impossibilité d'un

9. Les territoires d'outremer gardent la possibilité d'introduire des restrictions au commerce avec les Six pour protéger les industries naissantes. Naturellement, de telles restrictions doivent concerner tous les pays membres du Marché commun, métropole incluse.

10. Cf. R. GIRAULT, La France entre l'Europe et l'Afrique, in: E. SERRA, Le relance européenne et les traités de Rome. Actes du colloque de Rome, 25-28 mars 1987, Giuffré, 1989, Milano, pp.351-378. Cf. aussi P. GUILLEN, L'avenir de l'Union française dans la négociation des traités de Rome, in: Relations internationales, 57(1989), pp.103-112; R. SCHREURS, L'Eurafrique dans les négociations du Traité de Rome 1956-1957, in: Politique africaine, 49(1993), pp.82-92; G. MIGANI, L'association des TOM au Marché Commun: histoire d'un accord européen entre cultures économiques différentes et idéaux politiques communs, 1955-1957, in: G. BOSSUAT, M-T. BITSCH (éds.), L'Europe unie et l'Afrique. De l'idée d'Eurafrique à la Convention de Lomé I. Actes du colloque de la Sorbonne, avril 2004, Bruylant, Bruxelles, 2005.

11. Archives MAEF, De-Ce, (Commissions économiques internationales), dossier 315, Note, 10 novembre 1958. Les Cinq sont tout à fait d'accord avec Paris sur ce point. Dans la note rédigée à la fin de la réunion du Conseil il est affirmé: «Il est à craindre qu'à l'occasion de discussions générales ne soit évoqué le problème de l'Association des pays et territoires d'outremer à la CEE. En effet, étant donné la prépondérance des pays indépendants, membres du Commonwealth et des territoires britanniques au sein de cette Commission, il est à présumer que les conséquences du Traité de Rome seront évoquées par la Commission. Les attaques dont a été l'objet le régime d'Association, à Genève, ainsi qu'à d'autres occasions, ne manqueront pas de se renouveler sur ce forum». AHUE, CM/1959, dossier 1026, Note du Secrétariat du Conseil de la CEE, 8 décembre 1958. 
développement économique des pays associés, producteurs de matières premières et de produits agricoles, dans le cadre d'une association avec des économies hautement industrialisées sans protection particulière. ${ }^{12}$

L'objectif des Six est d'obtenir le statut d'observateur pour le représentant de la CEE. A titre provisoire, il est envisagé d'accepter une invitation personnelle adressée à la Commission européenne par le Secrétaire exécutif. Une délégation des Six prendra les contacts nécessaires avec le Secrétaire Exécutif de la CEA

«afin d'examiner avec celui-ci la possibilité d'obtenir une invitation officielle en faveur de la Communauté. Au cas où la réponse du Secrétaire Exécutif serait favorable, la Commission [européenne] en serait avisée immédiatement par télégramme pour déléguer, dans les plus brefs délais, un représentant à Addis Abeba». ${ }^{13}$

Comme les Six s'y attendaient, la première session de la CEA est l'occasion pour les Etats africains de manifester leur mécontentement à l'égard du régime d'association. ${ }^{14}$ Malgré toutes les argumentations préparées par les Six et la Commission, ${ }^{15}$ la France, la Belgique et l'Italie sont impuissantes face à des pays africains bien déterminés à refuser à la CEE un statut d'observateur. En revanche, ces derniers sont favorables à l'établissement de relations avec la CCTA, le Comité Economique de la Ligue arabe et la Conférence d'Accra, (qui regroupe les Etats indépendants d'Afrique). La seule concession obtenue par les Six est que le Secrétaire exécutif pourra

«soit de sa propre initiative, soit à la demande d'un membre, porter à la connaissance de la [CEA] des renseignements détaillés sur toute autre organisation intergouvernementale fonctionnant en Afrique, avec laquelle la Commission pourrait avoir le désir d'établir des relations similaires». ${ }^{16}$

Pour éviter un vote défavorable qui pourrait être durablement préjudiciable aux intérêts des Six, la France, la Belgique et l'Italie préfèrent ne pas proposer d'inclure la CEE dans la liste des organisations avec lesquelles la CEA devra établir des liens. Pour exprimer leur désapprobation les trois délégations se bornent à s'abstenir lors du vote. De plus, elles décident de ne pas s'opposer à une résolution, avancée par l'Ethiopie, ${ }^{17}$ qui invite le Secrétaire exécutif à entreprendre une étude sur les incidences de la constitution de la CEE sur les économies africaines. Les trois délégations se contentent d'exprimer le souhait que l'étude en question utilise les travaux déjà effectués par d'autres organisations internationales, notamment par le GATT.

12. AHUE, CM/1959, dossier 1026, Ministère français des Affaires Etrangère, Note, 6 décembre 1958.

13. AHUE, CM/1959, dossier 1026, Note introductive, 17 décembre 1958.

14. Addis Abeba sera choisie comme siège officiel de la Commission.

15. AHUE, CM/1959, dossier 1026, Annexe I à la Note introductive, 17 décembre 1958.

16. AHUE, CM 2/1959, dossier 1026, Résolution adoptée par la Commission à sa septième séance, le $1^{\text {er }}$ janvier 1959. Annexe à la Note d'information, 16 janvier 1959.

17. «L'attitude de l'Ethiopie semble s'expliquer par les incidences du Traité de Rome sur les exportations de café éthiopien, ainsi que par le fait que ce pays, n'appartenant pas au GATT, n'a pas pu encore exprimer ses appréhensions sur le plan international». Archives MAEF, De-Ce (Commissions économiques internationales), dossier 315, Note, 14 janvier 1959. 


\section{La polémique contre la CEE et le régime d'association s'approfondit}

A la fin de la session de 1959, si la coordination entre les membres de la CEE a été effective, les Six n'ont pas atteint leurs objectifs: non seulement la CEE n'a pas un statut d'observateur, mais son rôle en Afrique reste très fortement contesté.

En effet, les Etats africains s'attaquent à la CEE pour des raisons économiques et politiques: d'un côté, ils estiment que les préférences accordées aux territoires d'outremer gêneront les exportations des pays tiers; de l'autre, le régime d'association représente à leurs yeux un obstacle pour l'unité économique et politique du continent. La polémique sur la CEE au sein de la CEA est très proche de celle développée au GATT. Cependant, tandis que dans l'organisation économique internationale les pays membres se focalisent sur les raisons économiques, au sein de la CEA les raisons politiques ont autant d'importance. ${ }^{18}$ Par ailleurs, il ne faut pas oublier que, pour la première fois, les Etats africains sont majoritaires dans un organisme international. Ils sont bien décidés à utiliser leur force numérique pour imposer leurs vues aux puissances européennes. Etant donnée la situation, il n'est pas étonnant que

«parlant de l'atmosphère générale, [le délégué français à Addis Abeba] souligne que les Etats indépendants d'Afrique ont entendu affirmer qu'un rôle prédominant leur revenait dans la CEA. Ils ont constamment tenu à montrer, par leur attitude et leurs propos, que les puissances européennes ne pouvaient participer à la CEA qu'en qualité de membres moins importants qu'eux-mêmes». ${ }^{19}$

Cependant, il ne s'agit pas seulement de s'imposer face aux puissances coloniales. Les pressions des pays africains visent aussi les territoires associés à la CEE. En s'attaquant aux modalités de l'association, les Etats africains font appel au désir d'unité et d'indépendance qui est très répandu parmi les populations des TOM. Même si cette recherche d'intégration ne se fait pas sans beaucoup d'arrière-pensées, le mythe de l'unité africaine conditionne fortement la politique étrangère de ces Etats. Dans ce contexte, l'idée d'Eurafrique n'a plus la même valeur. ${ }^{20}$ Pour les pays de l'Afrique anglophone, elle n'a aucune signification; pour certains leaders, l'Eurafrique est seulement une justification idéologique de la

18. Selon le Conseil de la CEE, «les Etats indépendants d'Afrique étaient animés par des motifs politiques et confondaient volontairement la sympathie à l'égard d'une organisation avec la reconnaissance de ces activités. Prétendant être mal renseignés sur la CEE (en dépit de la diffusion de la note établie par la Commission de la CEE) ils ont déclaré vouloir admettre au bénéfice de ces relations une liste d'organisation qui: [...] i) incluait, outre la CCTA, le Comité Economique de la Ligue arabe et la Conférence d'Accra; ii) excluait la CEE. Le Maroc et la Tunisie ont même menacé, au cas où les puissances occidentales insisteraient pour mettre la CEE sur le même pied que les trois premières Organisations, de présenter et de faire admettre la candidature du Secrétariat du Maghreb uni (organisation que son caractère non gouvernemental aurait dû suffire à écarter). Ils ont affirmé que par 'esprit de compromis' ils consentaient à ne pas présenter la candidature de cette dernière organisation si celle du Marché commun était abandonnée». AHUE, CM 2/1959 dossier 1026, Note d'information, 16 janvier 1959.

19. AHUE, CM 2/1959, dossier 1026, Extrait du procès-verbal de la $41^{\text {ème }}$ réunion du COREPER, 16 janvier 1959.

20. Sur la question voir G. BOSSUAT, M-T. BITSCH (éds.), L'Europe unie et l' Afrique ..., op.cit. 
domination coloniale. En fait, ils estiment que l'association des TOM à la CEE empêche ces derniers d'exercer leur pleine indépendance.

Le projet des Etats africains indépendants, ou au moins d'une partie importante d'entre eux, est la création d'une organisation politique panafricaine et d'un marché commun. Avec l'aide des Nations Unies, les pays africains espèrent promouvoir leur développement et accéder à un statut de puissance internationale. ${ }^{21}$ A Paris, où on regarde avec beaucoup de méfiance les activités de la CEA, le Quai d'Orsay décrit en ces termes les objectifs des pays africains membres de la CEA, ou au moins de certains d'entre eux:

«Tentative de rupture des liens institutionnels entre une partie de l'Afrique et une partie de l'Europe (liens correspondant à la Communauté française et à la CEE), exaltation de solidarités exclusivement africaines, utilisation de l'ONU pour parvenir à ces deux objectifs et se prémunir contre les dangereuses séductions de l'aide bilatérale, telle semble être la politique principale des membres activistes de la CEA». ${ }^{22}$

Face aux critiques, la réaction des Six est de coordonner plus étroitement leur attitude. Avant la deuxième session de la CEA, prévue pour janvier 1960, ils tiennent plusieurs réunions afin de définir une position commune. Ils décident que la CEE n'aura pas à demander un statut d'observateur:

«Le prestige de la Communauté [...] d'une part, et d'autre part, le fait que l'existence des rapports entre la CEE et la CEA découle de la nature des choses et présente un intérêt réciproque pour ces deux organisations, doivent interdire à la Communauté de se présenter dans la position de demandeur». ${ }^{23}$

Par ailleurs, un document, rédigé par la Commission, résume les observations que le porte-parole des Six pourra avancer lors du débat sur le Marché commun. Celles-ci concernent le fait que le rapport se fonde sur des données statistiques anciennes, il examine les effets de la création de la CEE sur les seuls pays africains non associés, et il a été élaboré sans avoir consulté les services de la CEE. D'autre part, il est affirmé que la CEE envisage avec faveur la création d'un ensemble économique africain, qui ne pourra avoir que des effets positifs sur le commerce et sur le développement du continent. Quant à la crainte de voir se fermer les marchés des Six, la production des TOM est largement insuffisante pour satisfaire la demande existante et, d'ailleurs, les TOM ne peuvent pas augmenter leur production de façon illimitée. Par conséquent, les importations en provenance des pays non associés seront toujours nécessaires. Enfin, les Six tiennent à rappeler que la CEE se propose de suivre une politique commerciale libérale: la Communauté s'est déclarée prête à négocier le niveau du TEC en acceptant les propositions du secrétaire d'Etat américain avancées dans le cadre du GATT.

21. A ce propos le jugement français est très clair: «... il est apparu nettement que les Etats africains considéraient que l'association des PTOM à la CEE empêchait la conquête par ces derniers de leur indépendance réelle et prévenait, plus encore, l'établissement d'une Communauté africaine, instrument jugé par eux indispensable pour parvenir à la puissance internationale». Archives MAEF, De-Ce (Commissions économiques internationales), dossier 316, Note, 9 février 1960.

22. Ibid.

23. AHUE, BAC 26/1959, dossier 524, Note d'information, 16 janvier 1960. 
La deuxième conférence de la CEA s'ouvre à Tanger le 26 janvier 1960. Les Français sont tout de suite placés dans une position difficile. Le Maroc, appuyé par le Ghana et la Guinée, conteste le droit de la délégation française à parler au nom de la Communauté franco-africaine. Les pays africains proposent l'adoption d'une résolution qui recommande aux puissances coloniales de consulter les territoires africains pour savoir s'ils veulent être associés à la CEA. Les territoires africains sous la tutelle de la Grande Bretagne et de la Belgique étant déjà associés à la Commission, la résolution vise essentiellement la France. En effet, malgré les explications juridiques du représentant français, celui-ci est sur la défensive et obligé d'assumer une position négative et tout à fait isolée. ${ }^{24}$

Le débat sur la CEE montre bien que la méfiance à l'égard du Marché commun n'est pas moindre. Les pays africains se refusent à admettre un représentant de la CEE à titre d'observateur. Ils considèrent que l'association des territoires africains au Marché commun européen non seulement empêche ces derniers de gagner une véritable indépendance, mais constitue aussi un obstacle à l'établissement d'une Communauté africaine.

Pendant la discussion sur le rapport concernant les conséquences du Marché commun sur les économies africaines, le Nigeria, le Ghana, la Tunisie et le Maroc déclarent que les préférences accordées aux pays associés stimuleront la production de façon anormale. Les explications de la Belgique et de la France - un marché européen plus vaste achètera plus de produits ${ }^{25}$ - ne sont pas suffisantes pour rassurer les délégations africaines. A la fin de la session, celles-ci approuvent une résolution pour poursuivre les études sur les groupements économiques en Europe.

\section{0-1963: la CEA s'élargit. Les nouvelles priorités de l'organisation}

L'année 1960 représente un moment important pour la CEA. 17 pays africains deviennent indépendants ${ }^{26}$ et entrent dans la Commission; surtout, presque tous les

24. Le représentant des Etats-Unis, venu en observateur, écrit au Département d'Etat à la fin de la conférence: «The fact that British and the Belgians had both put forward African territories for associate membership status did not enhance the French position. France was put on the defensive and forced to assume negative positions. This was easily achieved and maintained despite the able representation of Mr. Gabriel Lisette, Vice Premier of the Republic of Chad and head of the French Delegation». NARA, RG 50, Central Decimal File, 1960-1963, File 342.70/1-660, Report of the US observer to the Second session of the ECA Tangier, Morocco.

25. Le représentant des Etats-Unis remarque que: «The argument that economic expansion in the EEC would broaden the market for all was not convincing to the African states because of the stress France and Belgium placed on the primarily political aims of the EEC and the priority 'naturally' given to the economic development of the associated territories». NARA, RG 50, Central Decimal File, 1960-1963, File 342.70/1-660, Report of the US observer to the Second session of the ECA Tangier, Morocco.

26. Côte d'Ivoire, Sénégal, Mali, Mauritanie, Dahomey (aujourd'hui: Benin), Haute Volta (aujourd'hui: Burkina Faso), Niger, Togo, Cameroun, Tchad, République Centrafricaine, Congo Brazzaville, Gabon, Madagascar, Congo Léopoldville (aujourd'hui: République démocratique du Congo), Nigeria, Somalie. 
nouveaux membres sont des Etats associés à la CEE. En même temps, l'Italie, une fois la Somalie indépendante, devient un simple observateur. Ne restent que la France et la Belgique pour représenter les Six.

Parmi les 17 pays africains qui accèdent à l'indépendance, 14 étaient des colonies françaises. Après le retour au pouvoir du général Charles de Gaulle, l'Union française avait été remplacée par la Communauté franco-africaine, un ensemble dominé par la France. Les Etats membres de la Communauté jouissaient d'une pleine autonomie intérieure, mais ils n'étaient pas indépendants. Soumise à différentes pressions, d'origine intérieure et extérieure, la Communauté disparait en 1961: le 16 mars un échange de lettres entre le Premier ministre français et le président du Sénat prend acte de la caducité du titre XII de la constitution, concernant les structures de la Communauté franco-africaine. Dorénavant les relations franco-africaines reposeront sur des traités de coopération bilatéraux, dont le but est d'organiser l'assistance économique, technique et militaire de l'ancienne métropole à ses ex-colonies. En même temps, après la proclamation de l'indépendance, les pays africains associés à la CEE confirment officiellement leur volonté de rester liés au Marché commun européen. ${ }^{27}$

L'adhésion des pays associés à la CEA implique un certain changement dans les équilibres de l'organisation. D'une part, la défense du régime d'association n'incombe plus aux seuls Etats européens; de l'autre, pour la première fois, les pays africains peuvent se consacrer concrètement à des projets de coopération économique et politique. En réalité, cette possibilité n'est pas immédiatement exploitée, parce que les Etats nouvellement indépendants tendent à se lier suivant les anciens clivages coloniaux. La méfiance est lente à disparaître entre les Etats arabes et les Etats de l'Afrique sub-saharienne. En outre, les pays africains sont partagés entre les modérés (ceux qui ont maintenu des liens avec les anciennes puissances coloniales) et les révolutionnaires, considérés comme les plus actifs dans la lutte contre l'impérialisme. ${ }^{28}$

En 1961, la discussion sur les effets du Marché commun sur les économies africaines se déroule de façon un peu différente. Elle voit la participation des Etats associés; un membre de la Commission est même invité à assister à titre officieux aux travaux des réunions préliminaires, qui auront lieu avant l'ouverture officielle de la session.

Les pays africains non associés articulent leurs observations de façon plus complexe. Ils présentent trois types de remarques: en premier lieu, il y a les soucis des pays dont les produits sont en concurrence avec les produits des pays associés. Ceux-ci craignent que les préférences tarifaires accordées aux territoires associés entraînent un accroissement de production plus rapide dans les pays associés que

27. Cf. G. MIGANI, La Francia e l' Africa sub-sahariana, 1957-1963: storia di una decolonizzazione fra ideali eurafricani e politica di potenza, Thèse de doctorat, Université de Florence-IEP de Paris, 2004.

28. Généralement, les pays membres du Groupe de Casablanca sont considérés comme 'révolutionnaires'. Il s'agit de la République Arabe Unie, le Ghana, la Guinée, le Mali, la Libye, le Soudan, le Maroc, et le Gouvernement provisoire de la République algérienne. 
dans les pays non associés. Ils s'inquiètent aussi des effets de la politique agricole commune sur leurs exportations. En deuxième lieu, il y a les critiques contre le régime d'association accusé d'être un obstacle majeur à l'industrialisation des pays associés, en raison d'une concurrence accrue des produits européens sur leurs marchés. Le Nigeria et la Tunisie tiennent à souligner qu'il importe de veiller à ce que les projets financés par le FEDOM apportent, en fin de compte, des avantages aux habitants des Etats associés. Enfin, il y a les observations de ceux qui considèrent que le régime d'association constitue une entrave à la création d'une solidarité exclusivement africaine. ${ }^{29}$ Certains pays n'hésitent pas à adopter un ton particulièrement virulent: la Guinée, par exemple, qualifie la CEE de

«force d'agression, de puissance néocolonialiste et de capitalisme qui exploite l'Afrique. En conclusion la délégation de la Guinée a déclaré textuellement que son Gouvernement refuse de s'associer à la CEE et a évoqué l'éventuelle création d'un marché commun africain en réaction au Marché commun européen». ${ }^{30}$

Face à ces remarques, les réponses de la Belgique, de la France et des pays associés ne se font pas attendre. Ces derniers avancent des arguments particulièrement intéressants, surtout parce que c'est la première fois qu'ils peuvent s'exprimer officiellement. Après avoir rendu hommage à l'idéal de l'unité africaine, ils affirment indispensable de privilégier une approche réaliste des problèmes économiques. ${ }^{31}$ Or, le régime d'association permet d'accroître leurs débouchés sur des marchés très importants et de bénéficier d'une aide financière substantielle. En outre, les faits ont montré que le régime d'association ne constitue pas un obstacle pour leur industrialisation ni pour le développement des échanges avec les autres pays africains. Enfin, la renégociation du régime d'association permettra de rechercher, d'un commun accord, les voies et les moyens d'une coopération mutuellement avantageuse. ${ }^{32}$ Pour conclure leur action, les pays associés présentent une résolution qui prévoit d'élargir les études, jusque là pratiquement limitées à la CEE, au Commonwealth et à l'Association européenne de libre échange.

29. Le délégué français résume certaines critiques: «Les délégués du Ghana, de la République Arabe Unie et du Soudan ont dénoncé les conséquences néfastes qu'entraîne à leur avis l'association de certains pays africains au Marché commun: dépendance vis-à-vis de l'Europe, impossibilité de diversifier les productions et notamment de développer l'industrie, division de l'Afrique, effets préjudiciables sur l'économie des pays non associés. Ils ont proposé l'élaboration d'une politique concertée de l'ensemble des Etats africains, notamment en ce qui concerne l'exportation des produits primaires. Sous une forme plus modérée les délégués du Nigeria et l'Ethiopie ont appelé eux aussi à l'unité de l'Afrique, insistant sur les dangers de sacrifier à des avantages immédiats les possibilités de développement économique à long terme». Archives MAEF, De-Ce (Commissions économiques internationales), dossier n.1073, Télégramme pour Paris, 25 janvier 1961.

30. AHUE, BAC 26/1969, dossier 524, Note d'Information, 27 février 1961.

31. «Le représentant du Gabon, tout en donnant son accord à l'idée d'une coopération africaine accrue [...] a marqué fortement qu'on ne pouvait sérieusement, à l'heure actuelle, substituer des débouchés proprement africains à ceux que des pays comme le sien trouvaient en dehors du continent et notamment en Europe». Archives MAEF, De-Ce (Commissions économiques internationales), dossier 1073, Note, 21 février 1961.

32. AHUE, BAC 26/1969, dossier 524, Note d'Information, 27 février 1961. 
Une fois achevé le débat sur la CEE, les discussions entrent dans une phase plus constructive. Les pays membres de la CEA tombent d'accord pour créer un Comité permanent du commerce (qui aura la tâche de poursuivre les travaux sur le Marché commun européen), une Banque africaine de développement et des bureaux sous-régionaux. Les délégations africaines soulignent la nécessité de stabiliser les prix des produits tropicaux et de trouver les moyens pour favoriser leur coopération économique. ${ }^{33} \mathrm{Il}$ est décidé de convoquer une réunion des pays producteurs africains qui, après s'être concertés, pourront entrer en rapport avec les producteurs d'autres régions ainsi qu'avec les pays consommateurs. ${ }^{34}$

Enfin, certaines délégations (y compris des représentants des Etats associés à la CEE) soutiennent un projet de résolution pour retirer aux Etats européens la qualité de membres de plein droit de la CEA. Le projet n'est pas voté mais il est représentatif d'une certaine attitude: les pays africains veulent s'organiser de manière autonome. Ils acceptent volontiers l'aide bilatérale, mais ils ne voient pas la raison d'impliquer les anciennes puissances coloniales dans un organisme chargé de réfléchir au développement de l'Afrique, et au renforcement des relations économiques entre les pays africains.

La session de 1962 voit les pays associés très impliqués dans la défense du régime d'association à la CEE. Engagés dans les négociations pour le renouvellement de la Convention d'association, ils tiennent à motiver leur position. Désormais en majorité, les pays associés s'organisent pour réfuter les critiques, «avec beaucoup de compétence» de l'avis du représentant français, et repoussent le texte sur la poursuite des études sur le Marché commun. ${ }^{35}$ En réalité l'action en faveur de la CEE des Etats associés n'implique pas, de leur part, une adhésion aux théories eurafricaines. L'association avec la CEE et le développement des échanges avec l'Europe ne sont pas une fin en soi. Quand les économies africaines seront suffisamment riches et industrialisées, les projets de coopération économique interafricaine auront la priorité. Une note à l'attention du président de la République française est très explicite à ce propos:

«si les Etats francophones ont, au cours des débats, défendu le régime d'association à la CEE, nombre d'entre eux paraissent estimer que cette association sera transitoire et devra disparaître le jour de la création d'un Marché commun africain. Tous se sont

33. «Ce thème a constitué l'un des leitmotiv des interventions des délégations africains, encore que les voies et moyens de cette coopération n'aient pas été précisés de manière concrète». AHUE, BAC 26/1969, dossier 524, Note d'Information, 27 février 1961.

34. AHUE, BAC 26/1969, dossier 524, Note d'Information, 27 février 1961.

35. «Ce dernier refus est évidemment très symptomatique de l'agacement que ressentent les Associés à l'égard de ces études continuelles. Il doit être noté cependant que, selon la remarque faite en séance par le Secrétaire exécutif, la résolution de l'an passé sur la poursuite des études sur le Marché commun est toujours en vigueur. [...] le rejet du texte sur la poursuite des études doit donc être interprété comme une indication de tendance que comme une décision empêchant les études en question d'être faites». Archives MAEF, De-Ce (CEE-Euratom, 1961-1966), dossier 1459, Note, 13 mars 1962. 
associés aux condamnations portées contre le capitalisme étranger; tous se sont réclamés d'un socialisme au contenu imprécis». ${ }^{36}$

D'autre part, les Etats associés, comme les Etats africains non associés, semblent considérer normal l'éviction des puissances européennes des organismes africains de coopération technique. En février 1962, le Portugal (avec l'Afrique du Sud) est exclu de la CCTA, tandis que le statut de la France, de la Grande Bretagne et de la Belgique est modifié: «D'ores et déjà il est prévu que les trois derniers membres 'blancs' n'auront plus le droit de vote au sein de la CCTA». ${ }^{37}$ Au sein de la CEA, les pays africains approuvent une résolution aux termes de laquelle la Commission recommande au Comité économique et social de l'ONU de décider:

- l'exclusion de l'Espagne et du Portugal;

- le passage de tous les autres Etats européens, ayant la responsabilité de territoires non autonomes situés en Afrique, de la catégorie de membres de plein droit à celle de membres associé; ${ }^{38}$

- l'inclusion automatique dans cette même catégorie de tous les territoires non autonomes d'Afrique.

L'africanisation progressive de la Commission semble tellement inévitable que le représentant des Etats-Unis à la CEA s'étonne de l'attitude des puissances européennes, qui attendent de voir leur statut progressivement réduit une année après l'autre: «the British and French had been foolish not to beat the Africans to the punch by offering to go into observer or associate member status before the question was raised by the Africans». ${ }^{39}$ Elles pourraient prendre directement l'initiative d'une telle décision, comme cela avait été le cas pour la CCTA.

Après 1962, l'activité de la CEA se concentre dans les différents comités spécialisés créés en son sein. La Commission consacre une bonne partie de ses énergies à la mise en place d'une Banque africaine de développement et d'un Institut africain de développement, qui verront le jour entre 1963 et 1964.

En 1963, la création de l'Organisation de l'Unité Africaine (OUA), première organisation panafricaine à caractère politique, représente un moment majeur. Si l'efficacité de la nouvelle organisation internationale laisse beaucoup à désirer, son importance est essentiellement symbolique. Pour les Etats africains, l'OUA n'est que le début d'un processus d'intégration politique et économique. S'il est vrai que

36. CHAN, Fonds public Foccart, dossier 104, Note à l'attention du Président de la République, 27 mars 1964.

37. CHAN, Fonds public Foccart, dossier 1409, Synthèse des évènements politiques concernant les Etats africains et malgache, février 1962.

38. «French Del. Dupraz made it clear during corridor discussions that while right to vote in UNECA was basically matter of indifference, slap against France was bound to give further impetus to 'cartierisme' and therefore involved risk that French aid might be reduced. After vote he said to us that, speaking personally, he felt France is better off in status of associate member (i.e.) with right to participate in debates but without vote (but that he was sure de Gaulle would not take kindly to this UNECA action)». NARA, RG 50, Central Decimal File, 1960-1963, File 342.70/11-361, Airgram from Addis Ababa, February 26, 1962.

39. NARA, RG 50, Central Decimal File, 1960-1963, File 342.70/3-162, Airgram from USUN, March 2, 1962. 
les pays africains envisagent leur intégration suivant des modalités différentes, la force d'attraction de l'idée d'unité politique et économique du continent reste intacte.

Même les pays associés considèrent leurs liens avec la CEE comme transitoires et subordonnés à la réalisation d'un marché commun africain. Le fait que, pendant les sessions de la CEA, ils refusent de participer aux réunions de coordination avec les Six, alors qu'ils partagent avec eux l'objectif de défendre le régime d'association, est plutôt indicatif. Ils craignent d'être vus comme trop proches des puissances européennes et d'être accusés de trahir la solidarité africaine. ${ }^{40}$ A ce égard, on peut rappeler qu'ils tiennent à insérer dans la Convention de Yaoundé un article affirmant que la convention ne sera pas un obstacle pour la conclusion d'unions douanières ou de zones de libre échange entre pays associés ou pays associés et Etats tiers. ${ }^{41}$

De leur côté, les Six suivent les efforts de coordination des pays africains, et, dans une certaine mesure, ils sont disposés à les soutenir. La seule exception partielle est la France, pour laquelle les idées d'Eurafrique et de Communauté franco-africaine continuent de garder une importance substantielle. ${ }^{42}$ En effet, une fois les pays africains devenus indépendants, la politique d'association revêt une autre signification. Il ne s'agit plus de soulager, au moins en partie, les charges économiques que supportent les Métropoles en faveur de leurs territoires, mais de mettre en œuvre une véritable politique d'aide au développement. ${ }^{43}$

40. Dans un contexte où «il s'agit d'être aussi ‘Africains' que possible, les liens [que les Etats africains francophones] maintiennent avec la France et avec les Six sont considérés par eux comme un motif de gêne et d'inhibition [...]. Ainsi, en dépit des difficultés qu'elle éprouve à se traduire dans les réalités économiques actuelles, l'idée de groupement panafricain est suffisamment forte pour que chaque orateur africain soit tenu de lui rendre ouvertement hommage et soit contraint, en quelque sorte, de s'excuser s'il paraît lui être infidèle par quelque association étroite avec des puissances non africaines. Peut-être y a-t-il là beaucoup de verbalisme dans un continent et une organisation qui donnent assez dans ce travers. Mais ce complexe de culpabilité chez ceux qui ne rompent pas leurs liens avec l'Europe ne saurait se maintenir sans grave dommage pour les solidarités qu'il affecte». Archives MAEF, De-Ce (Commissions économiques internationales), dossier 1073, Note, 21 février 1961.

41. Art. 8 et 9 de la Convention. Cf. aussi N. DELORME, L'Association des Etats africains et malgache à la CEE, LGDJ, Paris, 1972, p.61.

42. D'ailleurs le représentant français avait rappelé aux pays membres de la CEA que «la France entendait trouver dans le régime d'association la synthèse des solidarités qui la liaient à une partie de l'Europe et à une partie de l'Afrique. De plus il avait insisté sur le fait que le régime contenu dans le Traité de Rome, loin de diviser l'Afrique, créait au contraire un espace économique africain et eurafricain d'une ampleur encore non connue par l'histoire, offrant aux jeunes industries africaines tous les débouchés souhaitables». Archives MAEF, De-Ce (Commissions économiques internationales), dossier 1073, Note, 21 février 1961.

43. «La CEE estime que sa politique d'association peut s'inscrire en Afrique dans une politique d'aide aux pays en voie de développement. Plutôt que de disperser ses moyens d'action, elle a préféré au moins dans un premier temps, les concentrer sur une zone où des liens déjà éprouvés lui rendaient la tâche plus aisée, où aussi sa situation sur les plans culturels et économiques lui créait des obligations». AHUE, BAC 26/1959, dossier 524, Annexe II à la Note d'information, 16 janvier 1960. Cf. aussi E. GRILLI, The European Community and developing countries, Cambridge University Press, Cambridge, 1993. 


\section{Conclusion}

Si au moment de sa création la CEA constitue, pour les Etats africains, le lieu où faire pression sur les puissances européennes pour les amener à accorder l'indépendance aux territoires africains, au fur et à mesure que les membres de la Commission augmentent, elle devient un espace privilégié de contacts entre les pays francophones et anglophones. Au sein de la CEA ceux-ci discutent du développement de l'Afrique, élaborent des projets de coopération régionale pour favoriser l'industrialisation du continent et pour stabiliser le prix des produits tropicaux. Ils s'emploient également à mettre en place une Banque de développement et un Institut de développement.

Dans ce contexte, la CEE doit d'abord se défendre de l'accusation de diviser l'Afrique. Sa tâche devient plus facile au fur et à mesure que les Etats associés deviennent membres de la Commission. Toutefois, les Six cherchent aussi à mettre en valeur leur action auprès des pays africains. En effet, à un moment où le poids du Tiers Monde - et de l'Afrique - s'accroît dans le système international, la définition d'une politique d'aide au développement est devenue une question essentielle pour les pays industrialisés. D'ailleurs Washington exerce beaucoup de pression sur ses alliés pour qu'ils augmentent les sommes consacrées à l'aide au Tiers Monde. ${ }^{44}$ Dans ce domaine, la CEE a un rôle à jouer. Son objectif principal est de favoriser le développement et l'industrialisation des pays associés, tout en les insérant dans les circuits d'échanges mondiaux. Même si ses moyens d'action sont concentrés dans une zone géographiquement limitée, elle tient à coopérer avec les organisations créées par les pays africains et à montrer, ainsi, son soutien à leurs efforts d'intégration économique. Pendant les années soixante, la Commission saura bâtir une politique de coopération dont l'importance ne cesse de grandir aux yeux des pays associés. En même temps, son influence augmente dans tout le continent africain. La Convention de Yaoundé, renouvelée en 1963 et en 1969, ainsi que la Convention de Lomé en 1975, représentent les instruments pour instaurer des relations nouvelles avec des pays en voie de développement.

44. Sur la politique d'aide américaine, voir G.M. GUESS, The politics of United States foreign aid, St. Martin's Press, New York, 1987; R.F. ZIMMERMAN, Dollars, diplomacy, and dependency: dilemmas of US economic aid, Rienner, Boulder, Colo, 1993; Y. BAUMANN, John Fitzgerald Kennedy und "foreign aid": die Auslandshilfepolitik der Administration Kennedy unter besonderer Berücksichtigung des entwicklungspolitischen Anspruchs, Franz Steiner Verlag, Stoccarda, 1990; S.H. BUTTERFIELD, U.S. development aid, an historic first: achievements and failures in the twentieth century, Praeger Publishers, Westport, Conn., 2004. 